\title{
Intriguing Aspects of Non-innocent Ligands in Transition Metal Complexes
}

\author{
Michael B. Hall \\ Department of Chemistry, Texas A\&M University, \\ College Station, TX 77843-3255 USA
}

Non-innocent ligands play important roles in a variety of catalytic reactions. However, the ambiguous nature of their electronic structure causes problem for the assignment of the metal's oxidation state and for a clear-cut role of the metal in their reaction mechanisms. Nickel bis(dithiolene) complexes, which exist in three oxidation states $\left[\mathrm{Ni}\left(\mathrm{S}_{2} \mathrm{C}_{2} \mathrm{R}_{2}\right)_{2}\right]^{\mathrm{n}}(\mathrm{n}=2-, 1-, 0)$, are all square-planar $\mathrm{Ni}(\mathrm{II})$ with redox active ligands. The neutral complex reacts with an alkene to produce the cis-interligand adduct, which upon reduction releases the alkene.

Among the related ruthenium tris(thiolate) complexes, $\left[\mathrm{Ru}(\mathrm{dppbt})_{3}\right]^{\mathrm{n}}(\mathrm{n}=1-, 0,1+)$ $(\mathrm{dppbt}=$ diphenylphosphinobenzenethiolate), the most oxidized form also reacts with alkenes, a reaction attributed to thyil-diradical character involving two S atoms. Although it seems clear that $\left[\mathrm{Ru}(\mathrm{dppbt})_{3}\right]^{1-}$ is $\mathrm{Ru}(\mathrm{II})$ with three thiolate ligands, the nature of the most oxidized form, $\left[\mathrm{Ru}(\mathrm{dppbt})_{3}\right]^{1+}$ appears elusive. High-level ab initio methods predict its ground state electronic structure to be an open shell diradical singlet state with antiferromagnetic coupling between $(\mathrm{S}=1 / 2) \mathrm{Ru}(\mathrm{III})$ and $(\mathrm{S}=1 / 2) \mathrm{S} 3 \mathrm{p}_{\mathrm{z}}$, rather than the previous description based on a diradical state involving two $\mathrm{S} 3 \mathrm{p}$ orbitals. These new results provide an improved understanding of this system's experimental behavior.

Oxidizing alcohols by using water as the oxygen donor with liberation of dihydrogen represents a safe and clean process for such oxidations. Several recently reported $\mathrm{Ru}$ complexes involve non-innocent ligands. Density functional calculations show that the reaction of $\left[\mathrm{K}(\mathrm{dme})_{2}\right]\left[\mathrm{Ru}(\mathrm{H})\left(\operatorname{trop}_{2} \mathrm{dad}\right)\right]$ (trop $\mathrm{dad}_{2}$ 1,4-bis $(5 H$-dibenzo[a,d]cyclohepten-5-yl)1,4-diazabuta-1,3-diene and dme = dimethoxyethane) begins with the anionic ruthenium complex $\left[\mathrm{Ru}(\mathrm{H})(\text { trop2dad) }]^{-}\right.$and involves three steps: (1) dehydrogenation of methanol to formaldehyde, (2) formation of formic acid from formaldehyde and water, and (3) decomposition of the formic acid to carbon dioxide and hydrogen. The dehydrogenation of methanol occurs on the ligand with the ruthenium atom as a spectator, where the methanol's proton (on the oxygen atom) and its hydrogen (on the carbon atom) simultaneously move to the ligand's nitrogen and carbon atoms, respectively.

These complexes also exhibit some unusual static structural issues. For example, the neutral complex, $\mathrm{Ru}(\mathrm{dppbt})_{3}$, can act as an electrocatalyst for both hydrogen evolution or hydrogen oxidation. The interesting protonated intermediate, $\left[\mathrm{HRu}(\mathrm{dppbt})_{3}\right]^{+}$, has been studied by x-ray crystallography that shows a protonated S. However, DFT calculations and the experimental structure appear to disagree on which $\mathrm{S}$ is actually protonated. The neutral complex, $\mathrm{Ru}$ (trop2dad), is also unusual. The only structural details come from the NMR, which shows a spectrum compatible with a $\mathrm{C}_{2 \mathrm{v}}$ structure, but the DFT calculations show that this structure has two imaginary frequencies. 